\title{
COMPUTING THE LOCATION AND THE DIRECTION OF BIFURCATION
}

\author{
Philip Korman, Yi Li, and Tiancheng Ouyang
}

Dedicated to Louis Nirenberg on his 80th birthday

\begin{abstract}
We consider positive solutions of the Dirichlet problem $u^{\prime \prime}(x)+\lambda f(u(x))=0$ on $(-1,1), \quad u(-1)=u(1)=0$.

depending on a positive parameter $\lambda$. Each solution $u(x)$ is an even function, and hence it is uniquely identified by $\alpha=u(0)$. We present a formula, which allows to compute all $\alpha$ 's where a turn may occur, and then we give another formula, which allows to compute the direction of the turn. As an application, we present a computer assisted proof of the exact bifurcation diagram in case $f(u)$ is any cubic with real and distinct roots. Another application is a computer assisted proof of a conjecture by S.-H. Wang [10], related to gas combustion.
\end{abstract}

\section{Introduction}

We study exact bifurcation diagrams and exact multiplicity of the positive solutions to the Dirichlet problem

$$
\begin{aligned}
& u^{\prime \prime}(x)+\lambda f(u(x))=0 \text { on }(-1,1), \\
& u(-1)=u(1)=0
\end{aligned}
$$

depending on a positive parameter $\lambda$. We recall that solutions of (1.1) are even functions, with $u^{\prime}(x)<0$ for $x>0$, and hence any solution is uniquely identified by $\alpha=u(0)$, see [6]. Actually, even more is true: the value of $u(0)=\alpha$ uniquely identifies both $\lambda$ and $u(x)$, as follows easily by scaling $\lambda$ out of (1.1), and using uniqueness for initial value problems, see E. N. Dancer [4]. Hence the solution curves of (1.1) can be faithfully depicted by two-dimensional curves on $(\lambda, \alpha)$ plane. It is customary to refer to these curves as bifurcation diagrams. The Figure 1 below gives such a bifurcation diagram for a general cubic. The shape of any bifurcation diagram is determined by the turning points. We provide a

Received by the editors January 14, 2005.

1991 Mathematics Subject Classification. AMS subject classification: 34B15.

Key words and phrases. Bifurcation points, direction of the turn, exact multiplicity of solutions.

Korman is supported in part by the Taft Faculty Grant at the University of Cincinnati.

$\mathrm{Li}$ is supported in part by the Xiao-Xiang Grant at the Hunan Normal University, and by the Natural Science Foundation of China (10471052). 
necessary and sufficient conditon on $\alpha$ for the solution to be singular and thus a necessary condition for the turning point to occur:

$$
G(\alpha) \equiv F(\alpha)^{1 / 2} \int_{0}^{\alpha} \frac{f(\alpha)-f(\tau)}{[F(\alpha)-F(\tau)]^{3 / 2}} d \tau-2=0,
$$

with $F(u)=\int_{0}^{u} f(t) d t$. This formula can be used to compute numerically all turning points. At the turning points we are interested in the turning direction. We show that the curve tuns to the right in $(\lambda, \alpha)$ plane if

$$
D(\alpha) \equiv \int_{0}^{\alpha} f^{\prime \prime}(u)\left(\int_{u}^{\alpha} f(s) d s\right)\left(\int_{0}^{u} \frac{d s}{\left(\int_{s}^{\alpha} f(t) d t\right)^{3 / 2}}\right)^{3} d u<0,
$$

and the turn is to the left if the opposite inequality is true.

For an application of the above formulas, we turn to the long standing open problem of exact multiplicity in case of a cubic $f(u)$. We consider positive solutions in case $f(u)=(u-a)(u-b)(c-u)$, i.e. it is a cubic whose roots are three distinct positive constants $a<b<c$

$$
\begin{gathered}
u^{\prime \prime}(x)+\lambda(u(x)-a)(u(x)-b)(c-u(x))=0 \text { on }(-1,1), \\
u(-1)=u(1)=0,
\end{gathered}
$$

and $\lambda$ is a positive parameter. We wish to understand exactly how many solutions this problem has, and how these solutions are connected if one varies $\lambda$.

This problem was studied by J. Smoller and A. Wasserman [8], who attempted to solve the problem in general, and succeeded in solving it for $a=0$. Later S.-H. Wang [9] solved the problem under some restriction on $a$. Both papers used the phase-plane analysis. (We will see later on that the approach in [8] could not possibly cover the general case.) Then P. Korman, Y. Li and T. Ouyang [6], [7] used bifurcation theory to attack the problem, but again some restrictions were necessary (all of the above mentioned papers covered more general $f(u)$, behaving like cubic). We shall now recall the results of [7], and describe what is the desired optimal result.

By letting $u=c v$, we may assume that $c=1$, so that our nonlinearity is $f(u)=(u-a)(u-b)(1-u)$, with new $a$ and $b$, i.e. we consider

$$
\begin{gathered}
u^{\prime \prime}(x)+\lambda(u(x)-a)(u(x)-b)(1-u(x))=0 \text { on }(-1,1), \\
u(-1)=u(1)=0 .
\end{gathered}
$$

This substitution allows us to "compactify" the parameter set, since now $0<$ $a<b<1$. It is well-known that for existence of positive solutions it is necessary that $\int_{a}^{1} f(t) d t>0$, i.e. $\frac{1}{12}(a-1)^{3}(1+a-2 b)<0$, or

$$
0<a<b<\frac{1+a}{2} \text {. }
$$


We define the functions $F(u)=\int_{0}^{u} f(t) d t$ and $I(u)=f^{2}(u)-2 F(u) f^{\prime}(u)$, and the constants $\beta=\frac{1+b}{2}$ (the point where a straight line through the point $(a, 0)$ touches the graph of $y=f(u)$ for $u>a$, i.e. $\left.f^{\prime}(\beta)=\frac{f(\beta)}{\beta-a}\right)$, and

$$
\gamma=\frac{1}{3}\left(1+a+b+\sqrt{1-a+a^{2}-b-a b+b^{2}}\right)
$$

(the larger root of $f^{\prime}(u)=0$ ). Then the Theorem 3.1 in P. Korman, Y. Li and T. Ouyang [7] implies the following.

Theorem 1.1. Assume that either

$$
\int_{a}^{\gamma} f(t) d t \leq 0
$$

or

$$
I(\beta) \geq 0
$$

Then there exists a critical $\lambda_{0}$, such that the problem (1.5) has exactly one positive solution for $0<\lambda<\lambda_{0}$, exactly two positive solutions at $\lambda=\lambda_{0}$, and exactly three positive solutions for $\lambda_{0}<\lambda<\infty$. Moreover, all solutions lie on two smooth solution curves. One of the curves, referred to as the lower curve, starts at $(\lambda=0, u=0)$, it is increasing in $\lambda$, and $\lim _{\lambda \rightarrow \infty} u(x, \lambda)=a$ for $x \in(-1,1)$. The upper curve is a parabola-like curve with exactly one turn to the right. (See Figure 1 below for the bifurcation diagram.)

In Figure 1 we use $u(0)$, i.e. the $L^{\infty}$ norm, to identify the solution.

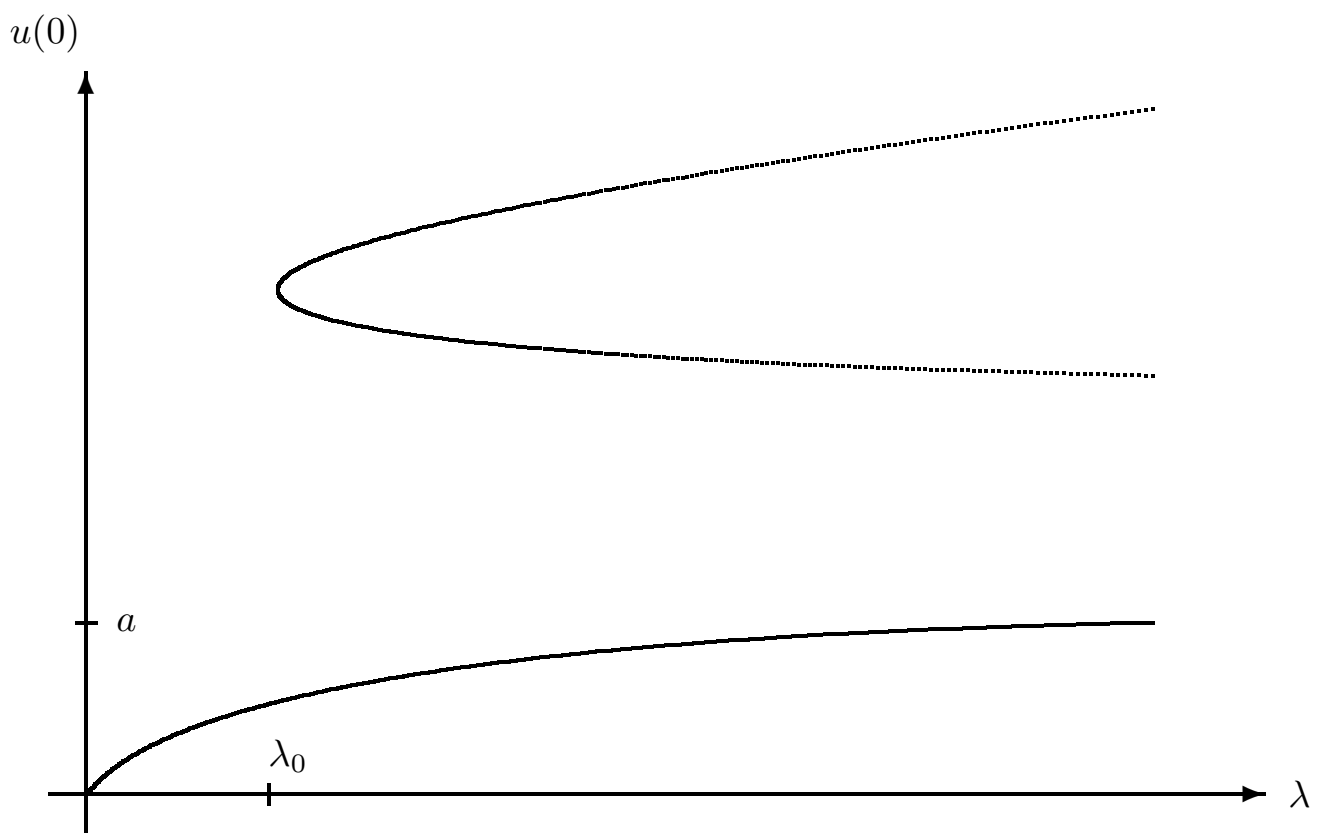

Figure 1 
What proportion of all possible $a$ and $b$ in the above conjecture is covered by our Theorem 1.1? In the $(a, b)$ plane the condition (1.6) defines a triangle $\Delta$ : $0<a<1, a<b<\frac{1+a}{2}$, whose area is $1 / 4$. Using Mathematica, we compute $I(\beta)=\frac{1}{384}(1-b)^{2}\left[24 a^{2}(b-1)^{2}-8 a(1+b)^{3}+(1+b)^{4}\right]$. So that the condition (1.8) holds if $g(a, b) \equiv 24 a^{2}(b-1)^{2}-8 a(1+b)^{3}+(1+b)^{4} \geq 0$. Similarly, the condition (1.7) holds if $h(a, b) \equiv \int_{a}^{\gamma} f(t) d t \leq 0$ (the function $h(a, b)$ is rather long). Using Mathematica, we now compute the area of the part of the triangle $\Delta$, call it $\Delta_{1}$, that satisfies either the inequality $g(a, b) \geq 0$ or $h(a, b) \leq 0$. Actually, we compute first the area of $\Delta \backslash \Delta_{1} \equiv \Delta_{2}$. Namely, after loading a package $\left(<<\right.$ Calculus $^{6}$ Integration $\left.^{6}\right)$, we give a command: Integrate[Boole $[a<b<$ $1 / 2+1 / 2 a \wedge h[a, b]>0 \wedge g[a, b]<0],\{a, 0,1\},\{b, 0,1\}]$ //N. Mathematica returns 0.146302 . The area of $\Delta_{1}$ is then 0.103698 . I.e. the Theorem 1.1 covers around 41.5 of all possibilities.

We now turn to the remaining 58.5. If $(a, b) \in \Delta_{2}=\Delta \cap\{g(a, b)<0\} \cap$ $\{h(a, b)>0\}$ the problem is open, but the results of P. Korman, Y. Li and T. Ouyang [6] still help. Indeed to justify the picture as in Figure 1, one needs to prove that only turns to the right are possible on the upper curve, and hence there is only one turn (the properties of the lower curve follow easily, since $f^{\prime}(u)<0$ for $0<u<a)$. Turns can only occur at singular $u(x)$, and it was proved in $[6]$ and [7], that $u(0)>\beta$ at any singular solution, and only the turns to the right are possible if $u(0)>\gamma$. This means there is a "danger" of a wrong turn only when $u(0)=\alpha \in(\beta, \gamma)$. The interval $(\beta, \gamma)$ tends to be small compared with $(\beta, 1)$. We see that in the full $(a, b, \alpha)$ parameter space the results of [6] and [7] actually cover the bulk of possibilities.

Recall that only the turns to the right are possible if the integral $D$, defined in (1.3), is negative. Hence, we would have a proof of the Theorem 1.2 below, if we could show that the "Calculus" integral $D$ is negative for $\alpha \in(\beta, \gamma)$. Unfortunately, this integral appears to be outside of the scope of Computer Algebra systems, like Mathematica or Maple. Numerical evaluation, and computer assisted proofs turn out to be quite feasible.

We provide a computer assisted proof, which shows that the conditions (1.7) and (1.8) are not necessary, thus giving a complete solution to the problem. The following is our main result.

Theorem 1.2. Assume that the necessary condition (1.6) holds. Then all conclusions of the Theorem 1.1 are true.

Our proof of this theorem is based on three independent numerical computations, each of which would constitute a "traditional" proof if their results could be analytically justified. Our first computation shows that in the "dangerous" region $\left((a, b) \in \Delta_{2}\right.$ and $\left.\alpha \in(\beta, \gamma)\right)$ the integral $D$, defined by (1.3) is negative. This implies that only turns to the right are possible on the upper curve, which justifies the Theorem 1.2. Our second computation shows that the function $G(\alpha)$ defined by $(1.2)$ is positive over $\Delta_{2} \times(\beta, \gamma)$. This means that no turns at all 
are possible in that region, which again implies that only turns to the right are possible on the upper curve. Our final computation uses a formula from phase plane analysis, and it shows that the curve travells to the left (in the $(\lambda, \alpha)$ plane) over $\Delta_{2} \times(\beta, \gamma)$, which leads to the same conclusion.

\section{Computing the direction of bifurcation}

We consider positive solutions of the Dirichlet problem

$$
u^{\prime \prime}(x)+f(u(x))=0 \text { on }(-1,1), u(-1)=u(1)=0 .
$$

It is well known that any solution $u(x)$ is an even function, with a unique point of maximum at $x=0$, and $u^{\prime}(x)<0$ on $(0,1)$. We assume that $u(x)$ is a singular solution of (2.1), i.e. the corresponding linearized problem

$$
w^{\prime \prime}(x)+f^{\prime}(u(x)) w=0 \text { on }(-1,1), w(-1)=w(1)=0
$$

admits a non-zero solution $w(x)$. It is also well known that $w(x)$ may be assumed to be positive, and it is an even function on $(-1,1)$, see [6]. For the singular solutions the following integral is important

$$
J \equiv-\int_{-1}^{1} f^{\prime \prime}(u(x)) w^{3}(x) d x=-2 \int_{0}^{1} f^{\prime \prime}(u(x)) w^{3}(x) d x .
$$

If $J \neq 0$ the critical point is non-degenerate, i.e. it persists under small perturbations of the equation in (2.1), see [4]. For the problem (1.1) depending on the parameter $\lambda$, the sign of $J$ determines the direction of bifurcation at the critical point in the $(\lambda, u(0))$ plane. If $J>0$ the curve turns to the right, and if $J<0$ to the left. We derive here a formula for $J$, which does not require a detailed knowledge of $u(x)$, and any knowledge of $w(x)$. It depends only on the maximal value of the critical solution $u(0) \equiv \alpha$.

Theorem 2.1. At any critical solution $u(x)$, with $u(0)=\alpha$,

$$
\begin{aligned}
J & =-c \int_{0}^{\alpha} f^{\prime \prime}(u)\left(\int_{u}^{\alpha} f(s) d s\right)\left(\int_{0}^{u} \frac{d s}{\left(\int_{s}^{\alpha} f(t) d t\right)^{3 / 2}}\right)^{3} d u, \\
& =-c D(\alpha),
\end{aligned}
$$

where $c=\frac{1}{4 \sqrt{2}} u^{\prime 3}(1) w^{\prime 3}(1)>0$.

Proof: Differentiate the equation (1)

$$
u^{\prime \prime \prime}(x)+f^{\prime}(u(x)) u^{\prime}(x)=0 \text { on }(-1,1) .
$$

Using this equation and (2.2), we conclude that the function

$$
u^{\prime \prime}(x) w(x)-u^{\prime}(x) w^{\prime}(x)
$$

is constant, and hence

$$
u^{\prime \prime}(x) w(x)-u^{\prime}(x) w^{\prime}(x)=-C, \quad \text { where } C=u^{\prime}(1) w^{\prime}(1)>0 .
$$


We rewrite this as

$$
\left(\frac{w}{u^{\prime}}\right)^{\prime}=\frac{C}{u^{\prime 2}}
$$

and then integrate, concluding that

$$
w(x)=-C u^{\prime}(x) \int_{x}^{1} \frac{1}{u^{\prime 2}(t)} d t .
$$

This formula will allow us to exclude $w(x)$ in $J$. (Observe that $\int_{x}^{1} \frac{1}{u^{\prime 2}(t)} d t$ tends to infinity as $x \rightarrow 0$, while $u^{\prime}(x)$ tends to zero. Hence both terms ought to be kept together in numerical computations.) Using (2.7) in ( 2.3)

$$
J=2 C^{3} \int_{0}^{1} f^{\prime \prime}(u(x)) u^{\prime 3}(x)\left(\int_{x}^{1} \frac{1}{u^{\prime 2}(t)} d t\right)^{3} d x
$$

We now wish to exclude $u^{\prime}(x)$ from (2.8). Since the energy $\frac{u^{\prime 2}}{2}(x)+F(u(x))$ is constant,

$$
\frac{u^{\prime 2}}{2}(x)+F(u(x))=F(u(0))=F(\alpha) .
$$

On the interval $(0,1)$ we express

$$
u^{\prime}(x)=-\sqrt{2} \sqrt{F(\alpha)-F(u(x))} .
$$

We use this formula in the integral $\int_{x}^{1} \frac{1}{u^{\prime}(t)^{2}} d t$, and then we make a change of variables $t \rightarrow s$, by letting $s=u(t)$. We have

$$
\begin{aligned}
\int_{x}^{1} \frac{1}{u^{\prime 2}(t)} d t & =-\frac{1}{2^{3 / 2}} \int_{x}^{1} \frac{u^{\prime}(t) d t}{[F(\alpha)-F(u(t))]^{3 / 2}} \\
& =-\frac{1}{2^{3 / 2}} \int_{u(x)}^{0} \frac{1}{[F(\alpha)-F(s)]^{3 / 2}} d s
\end{aligned}
$$

We then have

$$
J=c \int_{0}^{1} f^{\prime \prime}(u(x)) u^{\prime}(x)[F(\alpha)-F(u(x))]\left(\int_{0}^{u(x)} \frac{1}{[F(\alpha)-F(s)]^{3 / 2}} d s\right)^{3} d x
$$

with $c=\frac{1}{4 \sqrt{2}} C^{3}$. Finally, replacing $F(\alpha)-F(u)$ by $\int_{u}^{\alpha} f(s) d s$, making a change of variables $u=u(x)$, and writing $\alpha$ for $u(0)$, we obtain (2.4).

\section{Computing the bifurcation points}

In the previous section we computed the direction of turn, assuming that bifurcation occurs at $u(0)=\alpha$. We now provide a way to determine all possible $\alpha$ 's at which bifurcation may occur, i.e. the corresponding solution of (2.1) is singular. 
Theorem 3.1. A solution of the problem (2.1) with the maximal value $\alpha=u(0)$ is singular if and only if

$$
G(\alpha) \equiv F(\alpha)^{1 / 2} \int_{0}^{\alpha} \frac{f(\alpha)-f(\tau)}{[F(\alpha)-F(\tau)]^{3 / 2}} d \tau-2=0 .
$$

Proof: We need to show that the problem (2.2) has a non-trivial solution. As follows by (2.7) (or by direct verification) the function $w(x)=$ $-u^{\prime}(x) \int_{x}^{1} \frac{1}{u^{\prime 2}(t)} d t$ satisfies the equation in (2.2). Also $w(1)=0$. If we also have

$$
w^{\prime}(0)=0
$$

then since $u(x)$ is an even function, the same is true for $w(x)$ (by uniqueness for initial value problems), and hence $w(-1)=0$, which gives us a non-trivial solution of (2.2). Conversely, every non-trivial solution of (2.2) is an even function, and hence (3.2) is satisfied.

Using the equation in (2.1), we compute

$$
w^{\prime}(x)=f(u(x)) \int_{x}^{1} \frac{1}{u^{\prime 2}(t)} d t+\frac{1}{u^{\prime}(x)}
$$

Using the formula (2.10) from the previous section and the one right below it, we express

$$
\begin{aligned}
2^{3 / 2} w^{\prime}(x) & =\int_{0}^{u(x)} \frac{f(u(x))}{[F(\alpha)-F(\tau)]^{3 / 2}} d \tau- \\
& \frac{2}{[F(\alpha)-F(u(x))]^{1 / 2}} .
\end{aligned}
$$

If we try to set here $x=0$, then both terms on the right are infinite. Instead, we observe that

$$
\begin{gathered}
-\frac{2}{[F(\alpha)-F(u)]^{1 / 2}}=-\int_{0}^{u} \frac{d}{d \tau} \frac{2}{[F(\alpha)-F(\tau)]^{1 / 2}} d \tau-\frac{2}{F(\alpha)^{1 / 2}} \\
=-\int_{0}^{u} \frac{f(\tau)}{[F(\alpha)-F(\tau)]^{3 / 2}} d \tau-\frac{2}{F(\alpha)^{1 / 2}} .
\end{gathered}
$$

Using (3.4) in (3.3), we obtain

$$
2^{3 / 2} w^{\prime}(x)=\int_{0}^{u(x)} \frac{f(u(x))-f(\tau)}{[F(\alpha)-F(\tau)]^{3 / 2}} d \tau-\frac{2}{F(\alpha)^{1 / 2}} .
$$

The integral on the right is now non-singular as we let $x \rightarrow 0$. At $x=0$ we see that (3.2) is equivalent to (3.1). 


\section{A computer assisted proof}

We parameterize the solutions of (1.1) by their maximum value $\alpha=u(0)$. (Recall that the value of $\alpha$ uniquely determines the solution $(\lambda, u(x))$.) By rescaling $x$ we can transform the problem (1.1) into

$$
\begin{gathered}
u^{\prime \prime}(x)+f(u(x))=0 \text { on }(-1,1), \\
u(-T)=u(T)=0,
\end{gathered}
$$

with $T=\sqrt{\lambda}$. Observe that $\frac{d \lambda}{d \alpha}$ and $\frac{d T}{d \alpha}$ have the same signs. To prove the theorem it then suffices to show that $\frac{d T}{d \alpha}$ changes sign exactly once on the upper curve, i.e. $T(\alpha)$ has exactly one critical point.

We now recall the phase-plane approach taken by J. Smoller and A. Wasserman [8], and S.-H. Wang [9]. With $S(\alpha)=\sqrt{2} T(\alpha)$, one easily has (see $(2.10)$ )

$$
S(\alpha)=\int_{0}^{\alpha}[F(\alpha)-F(u)]^{-1 / 2} d u
$$

and then

$$
S^{\prime}(\alpha)=\frac{1}{2 \alpha} \int_{0}^{\alpha} \frac{\theta(\alpha)-\theta(u)}{[F(\alpha)-F(u)]^{3 / 2}} d u,
$$

where $\theta(u)=2 F(u)-u f(u)$. It was proved in [8] that

$$
S^{\prime \prime}(\alpha)+\frac{2}{\alpha} S^{\prime}(\alpha)>\frac{1}{2 \alpha^{2}} \int_{0}^{\alpha} \frac{\phi(\alpha)-\phi(u)}{[F(\alpha)-F(u)]^{3 / 2}} d u,
$$

with $\phi(u)=u \theta^{\prime}(u)-\theta(u)$. (This formula is numbered (2.6) in both [8] and [9].) If one could show that the integral on the right in (4.3) is positive for all $\alpha$, then clearly $S(\alpha)$ can have only one critical point, a point of minimum. This was the strategy pursued in [8]. However, our numerical computations show that this integral is sometimes negative. For example, this integral is negative if $a=0.77$, $b=0.87$ and $\alpha=0.94 \operatorname{sh} 4 . n b$ (recall that $c=1$ ). Hence, a proof along these lines is impossible.

One way we obtain a computer assisted proof is by showing that the function $S^{\prime}(\alpha) \equiv S_{\alpha}(\alpha, a, b)$ given by (4.2) is negative for all values of $a$ and $b$ not covered by the Theorem 1.1, and all $\beta<\alpha<\gamma$. This means that the solution curve travels to the left in the $(\lambda, \alpha)$ plane for these values of parameters $a, b$ and $\alpha$, while for any other admissible values of the parameters only turns to right are possible on the upper curve. Our computaions show that the maximum of $S^{\prime}(\alpha)$ on the region $\bar{\Delta}_{2} \times[\beta, \gamma]$ occurs around $a_{0}=b_{0}=0.192, \alpha_{0}=0.73$, with the maximum value around -0.037 . This value is close to zero, but definitely negative, even allowing for the computational errors. This computation by itself is sufficient for the proof, but for extra assurance we computed the integral $J$ defined by (2.4). Actually we have computed $I I \equiv-F(\alpha)^{5 / 2} \int_{0}^{\alpha} f^{\prime \prime}(u)\left(\int_{u}^{\alpha} f(s) d s\right)\left(\int_{0}^{u} \frac{d s}{\left(\int_{s}^{\alpha} f(t) d t\right)^{3 / 2}}\right)^{3} d u$, where the 
extra term $F(\alpha)^{5 / 2}$ is introduced to make this integral scaling invariant in $f$. (Other such factors can be chosen, this one is suggested by the value of $u^{\prime}(1)$.) Our computation shows that $I I\left(a_{0}, b_{0}, \alpha_{0}\right) \approx 4.97$, which implies that only turns to the right are possible near $\left(a_{0}, b_{0}, \alpha_{0}\right)$.

We have obtained even further assurance by computing the function $G(\alpha)$ from the Theorem 3.1. It turned out to be positive over the "dangerous" region $\bar{\Delta}_{2} \times[\beta, \gamma]$. This means that no turns happen in that region. Not surprisingly, the minimum value of $G(\alpha) \approx 0.013$ over that region occurs near the same $a_{0}=b_{0} \approx 0.192, \alpha_{0} \approx 0.73$ as before.

\section{Validating the bifurcation diagrams}

It is very easy to compute numerically bifurcation diagrams for the problem (1.1). Indeed, one solves the equation in (4.1) with the initial data $u(0)=\alpha>0$, $u^{\prime}(0)=0$, and calculates the first $T$ so that $u(T)=0$. Then we set $\lambda=T^{2}$ (recall the derivation of (4.1)). This gives us a point $(\lambda, \alpha)$ on the bifurcation diagram for the problem (1.1). Repeating this procedure for sufficiently many relevant $\alpha$ 's, we obtain a bifurcation diagram. In Mathematica this procedure requires only one simple loop containing a NDSolve command. The tools developed in this paper, particularly the formulas (2.3) and (3.1) will often allow one to prove correctness of the bifurcation diagram. We describe our approach next.

If $S^{\prime}(\alpha)<0$ the curve travels to the left at $u(0)=\alpha$ in $(\lambda, \alpha)$ plane, it travels to the right if $S^{\prime}(\alpha)>0$, and at the turning points $S^{\prime}(\alpha)=0$. If $S^{\prime}(\alpha)$ is close to zero we may be unable to decide which way the curve travels because of the errors and uncertainties of the computer implementation. Let $\epsilon>0$ be such that for $\left|S^{\prime}(\alpha)\right|>\epsilon$ we can prove that the observed (computed) sign of $S^{\prime}(\alpha)$ is correct. For such $\alpha$ 's we know for sure which way the curve travels. The "danger" is near the turning points, where $\left|S^{\prime}(\alpha)\right|$ is small. How do we know that there is only one turning point there, rather than 3, 5, etc? Let $L$ be an interval of $\alpha^{\prime}$ 's where $\left|S^{\prime}(\alpha)\right|<\epsilon$. Assume we can prove (through a computation supported by estimates) that the integral $J$, given by (2.4) is positive. Then only turns to the right are possible over $L$, and hence at most one turn is possible. If, moreover, $S^{\prime}(\alpha)<0$ to the left of $L$ and $S^{\prime}(\alpha)>0$ to the right of $L$ then exactly one turn to the right occurs on $L$.

In addition, we can use the formula (3.1) to show that the problem (1.1) does not have any turning points for some parameter regions. It appears that our tools are sufficient to validate any bifurcation diagram, where the turning points are all non-degenerate (i.e. $\left.\int_{-1}^{1} f^{\prime \prime}(u) w^{3} d x \neq 0\right)$.

Next we turn to a prominent example involving a degenerate critical point. The following problem, related to gas combustion,

$$
u^{\prime \prime}+\lambda e^{\frac{a u}{a+u}}=0 \quad \text { on }(-1,1), \quad u(-1)=u(1)=0
$$


has been extensively studied, see S.-H. Wang, [10] and P. Korman and Y. Li [5], where the references to some earlier work are given. We regard $a>0$ as a fixed parameter, and again draw bifurcation diagrams in $(\lambda, \alpha)$ space, where $\alpha=u(0)$. It is known that for $a \leq 4$, the solution curve is monotone in $\lambda$ (i.e. for every $\lambda>0$ there exists a unique positive solution), while for $a \geq a_{1} \approx 4.35$ the solution curve is exactly $S$-shaped, see [10] and [5]. It has been conjectured by S.-H. Wang [10] that there is a unique $a_{0}\left(a_{0} \in\left(4, a_{1}\right)\right)$, so that the solution curve of (5.1) is $S$-shaped for $a>a_{0}$, and the curve is monotone for $a \leq a_{0}$. Clearly, the turning point at $a_{0}$ would be a degenerate critical point, since it continues for $a>a_{0}$, but not for $a<a_{0}$. We recall from [5] that for any $a$ the solution curve starts at $(\lambda=0, u=0)$ and continues to $(\lambda=\infty, u=\infty)$.

Again, we reduce the problem to a computation over a compact range of parameters. Let $u_{2}=u_{2}(a)$ be the larger of two roots of the equation $u f^{\prime}(u)=$ $f(u)$, with $f(u)=e^{\frac{a u}{a+u}}, u_{2}=\frac{1}{2}\left(-(2-a) a+a^{3 / 2} \sqrt{a-4}\right)$. The function $f(u)=$ $e^{\frac{a u}{a+u}}$ is convex for $u<-a+a^{2} / 2$ and concave for $u>-a+a^{2} / 2$. It was proved in [5] that if we can find a $\beta>-a+a^{2} / 2$ such that $f^{2}(\beta)-2 F(\beta) f^{\prime}(\beta)>0$, then for any for $\alpha \geq \beta$ a turn to the right occurs at any singular solution, i.e. the singular solution is non-degenerate. Our first computation showed that we can take $\beta=8$, i.e. for all $a \in\left(4, a_{1}\right)$ we have $f^{\prime \prime}(8)<0$ and $f^{2}(8)-2 F(8) f^{\prime}(8)>0$. Hence, it suffices to understand the bifurcation diagrams for $4<a<a_{1} \approx 4.35$ and $0<\alpha<8$. Over this range we have computed the function $G(\alpha)$ given by (3.1). It turned out to be a unimodular function which is negative for $a$ close to 4 and it has exactly two roots for larger $a$, with the transition occuring slightly below 4.07. We have computed that over the above range of parameters, $\frac{\partial}{\partial a} G(\alpha)>0$ while $G^{\prime \prime}(\alpha)<0$. Hence for increasing $a$ there is a critical $a_{0}$ at which the number of roots of $G(\alpha)$ goes from exactly zero for $a<a_{0}$, to exactly one at $a=a_{0}$, and exactly two for $a>a_{0}$.

The solution curve has to be monotone for $a<a_{0}$. Indeed, in this case the curve can have at most one turning point (for $\alpha>8$ ), and since the solution curve joins $(\lambda=0, u=0)$ to $(\lambda=\infty, u=\infty)$, it can have no turns at all. We claim that the solution curve is monotone at $a=a_{0}$ too. Indeed, there is exactly one critical point for $\alpha \in(0,8)$, and no critical point for $\alpha>8$ (a critical point in the $\alpha>8$ range would have to be degenerate, since it does not continue for $a<a_{0}$, which is impossible). The solution curve has to become $S$-shaped immediately for $a>a_{0}$. Indeed, we know that it becomes $S$-shaped eventually. This transition requires a degenerate critical point, which according to the computations described above happens only at $a=a_{0}$. For the same reason the solution curve stays $S$ - shaped for all $a>a_{0}$. Hence our computations imply the proof of S. -H. Wang's conjecture.

\section{Proof of Theorem 1.1}

In this short section we sketch and clarify the proof of the Theorem 1.1 from [6] and [7]. The papers [6] and [7] gave two ways to reduce the proof of the Theorem 
1.1 to showing positivity of the integral $K \equiv \int_{0}^{1} f^{\prime \prime}(u(x)) u^{\prime}(x) w^{2}(x) d x$ at any critical solution $u(x)$, with $w(x)$ being a positive solution of the corresponding linearized problem. Two representations were derived for $K$ :

$$
K=\frac{1}{\lambda}{w^{\prime}}^{2}(1)-w^{2}(0) f^{\prime}(\alpha)
$$

with $\alpha=u(0)$, and

$$
K=\frac{w^{2}(0)}{2 F(\alpha)} I(\alpha)
$$

where as before $I(u)=f^{2}(u)-2 F(u) f^{\prime}(u)$. If the condition (1.7) holds, then it is easy to see that $\alpha>\gamma$, and hence $f^{\prime}(\alpha)<0$, which implies that $K>0$ by (6.1). So assume (1.8) holds. By above, it suffices to consider $\alpha<\gamma$. Since it was proved in [7] that $\alpha>\beta$ at any singular solution, we may assume that $\alpha \in(\beta, \gamma)$.

Case 1. Assume that $F(\beta) \geq 0$. Then $F(\alpha)>F(\beta)$ for all $\alpha>\beta$. Since $I(\beta) \geq 0$, and

$$
I^{\prime}(\alpha)=-2 F(\alpha) f^{\prime \prime}(\alpha)>0 \text { for } \alpha>\beta,
$$

it follows that $I(\alpha)>I(\beta)>0$, and from $(6.2), K>0$.

Case 2. Assume that $F(\beta)<0$. If $F(\alpha)<0$ for all $\alpha \in(\beta, \gamma)$, we see that $I>0$ directly from its definition, and hence $K>0$. Otherwise, we can find $\bar{\beta} \in(\beta, \gamma)$, so that $F(\bar{\beta})=0$ and $F(\alpha)>0$ for $\alpha>\bar{\beta}$. Then $I>0$ on $(\beta, \bar{\beta}]$ directly from the definition of $I$, while on the interval $(\bar{\beta}, \gamma)$ we repeat the proof from the Case 1 to show that $I>0$.

\section{Acknowledgments}

It is a pleasure to thank Dieter S. Schmidt for many stimulating discussions, and for making useful modifications in our Mathematica programs, and S.-H. Wang for useful comments.

\section{References}

[1] I. Addou, Multiplicity results for classes of one-dimensional p-Laplacian boundary-value problems with cubic-like nonlinearities, Electron. J. Differential Equations, no. 52, (2000).

[2] K.C. Chang, Morse theory on Banach space and its applications to partial differential equations, Chinese Ann. Math. Ser. B 4, no. 3, (1983) 381-399.

[3] _ Infinite-dimensional Morse Theory and Multiple Solution Problems. Progress in Nonlinear Differential Equations and their Applications, 6. Birkhuser Boston, Inc., Boston, MA, 1993.

[4] E. N. Dancer, On the structure of solutions of an equation in catalysis theory when a parameter is large, J. Differential Equations 37, (1980) 404-437.

[5] P. Korman and Y. Li, On the exactness of an S-shaped bifurcation curve, Proc. Amer. Math. Soc. 127, no. 4, (1999) 1011-1020.

[6] P. Korman, Y. Li and T. Ouyang, Exact multiplicity results for boundary-value problems with nonlinearities generalising cubic, Proc. Royal Soc. Edinburgh, Ser. A 126A, (1996) 599-616. 
[7] _ Perturbation of global solution curves for semilinear problems, Adv. Nonlinear Stud. 3 no. 2, (2003) 289-299.

[8] J. Smoller and A. Wasserman, Global bifurcation of steady-state solutions, J. Differential Equations 39, (1981) 269-290.

[9] S.-H. Wang, A correction for a paper by J. Smoller and A. Wasserman, J. Differential Equations 77, (1989) 199-202.

[10] _ On S-shaped bifurcation curves, Nonlinear Anal. 22, no. 12, (1994) 1475-1485.

[11] _ On the time map of a nonlinear two-point boundary value problem, Differential Integral Equations 7 (1994) 49-55.

[12] S.-H. Wang and N. D. Kazarinoff, Bifurcation of steady-state solutions of a scalar reaction-diffusion equation in one space variable, J. Austral. Math. Soc. Ser. A 52 (1992) 343-355.

Department of Mathematical Sciences, University of Cincinnati, Cincinnati Ohio 45221-0025

E-mail address: kormanp@math.uc.edu

Department of Mathematics, Hunan Normal University, Changsha 410081, Hunan, AND, UNIVERsity OF IOWA, IOWA CITy IOWA 52242

E-mail address: yli@math.uiowa.edu

Department of Mathematics, Brigham Young University, Provo, Utah 84602

E-mail address: ouyang@math.byu.edu 